\title{
Sister Mary Joseph Nodule in Advanced Pancreatic Adenocarcinoma Identified on ${ }^{18}$ F-FDG PET/MRI
}

\author{
Ming Yang ${ }^{1}$, Alix C. Hopp ${ }^{1}$, Tanios S. Bekaii-Saab ${ }^{2}$, and Joseph M. Collins ${ }^{1}$ \\ ${ }^{I}$ Department of Radiology, Mayo Clinic, Scottsdale, Arizona; and ${ }^{2}$ Department of Hematology/Oncology, Mayo Clinic, Scottsdale, \\ Arizona
}

Sister Mary Joseph nodule is an umbilical metastatic lesion typically originating from gastrointestinal or gynecologic malignancies. In pancreatic cancer, Sister Mary Joseph nodule is a sign of advanced disease with an associated poor prognosis. ${ }^{18} \mathrm{~F}-\mathrm{FDG} \mathrm{PET} / \mathrm{MRI}$ provides an imaging tool in the identification of Sister Mary Joseph nodule and helps to improve the staging of pancreatic cancer.

Key Words: molecular imaging; oncology: pancreas; PET/MRI; pancreatic cancer; Sister Mary Joseph nodule

J Nucl Med Technol 2019; 47:341-342

DOI: 10.2967/jnmt.119.225961

\section{O}

ster Mary Joseph nodule refers to a metastatic lesion of the umbilicus, with the primary malignancy originating in the gastrointestinal or genitourinary system. Pancreatic cancer accounts for 7\%-9\% of the reported cases of Sister Mary Joseph nodule, with nearly $90 \%$ of tumors occurring at the pancreatic body and tail (1-2).

${ }^{18}$ F-FDG PET/CT has been used to detect Sister Mary Joseph nodule, given its excellent staging ability (3). Here, we describe a Sister Mary Joseph nodule in advanced pancreatic cancer discovered on ${ }^{18}$ F-FDG PET/MRI. Combining PET molecular imaging and diagnostic abdominal MRI information, PET/MRI is a powerful imaging tool in the diagnosis and staging of pancreatic cancer.

\section{CASE REPORT}

A 75-y-old man with biopsy-proven pancreatic adenocarcinoma had a rising serum level of the tumor biomarker carbohydrate antigen 19-9, at 41,154 U/mL, after 1 cycle of modified FOLFIRINOX chemotherapy (folinic acid, fluorouracil, irinotecan, and oxaliplatin). Diagnostic wholebody CT performed at an outside institution revealed a new liver metastasis in addition to the known primary pancreatic body tumor. Restaging hybrid ${ }^{18}$ F-FDG PET/MRI, includ-

For correspondence or reprints contact: Ming Yang, Department of Radiology, Mayo Clinic, 13400 E. Shea Blvd., Scottsdale, AZ 85259.

E-mail: yang.ming@mayo.edu

Published online Apr. 24, 2019.

COPYRIGHT (c) 2019 by the Society of Nuclear Medicine and Molecular Imaging. ing a torso PET/MRI survey scan and focused 1-bed-position contrast-enhanced diagnostic pancreatic PET/MRI, was performed to reassess the tumor burden. On both the torso PET/MRI survey and the focused diagnostic pancreatic PET/MR images, a 2.3-cm hypermetabolic lesion with an MRI signal pattern of a solid, enhancing mass was identified at the retroumbilical region (Figs. 1 and 2). In retrospect, this mass lesion was present on the abdominal CT image but was diagnosed as umbilical fungal infection. The patient had been on $6 \mathrm{wk}$ of antifungal therapy for suspected periumbilical fungal infection, which had improved since the start of chemotherapy. An ultrasound-guided coreneedle biopsy was performed and confirmed metastatic pancreatic adenocarcinoma of the umbilicus, also known as a Sister Mary Joseph nodule. On the basis of this proof

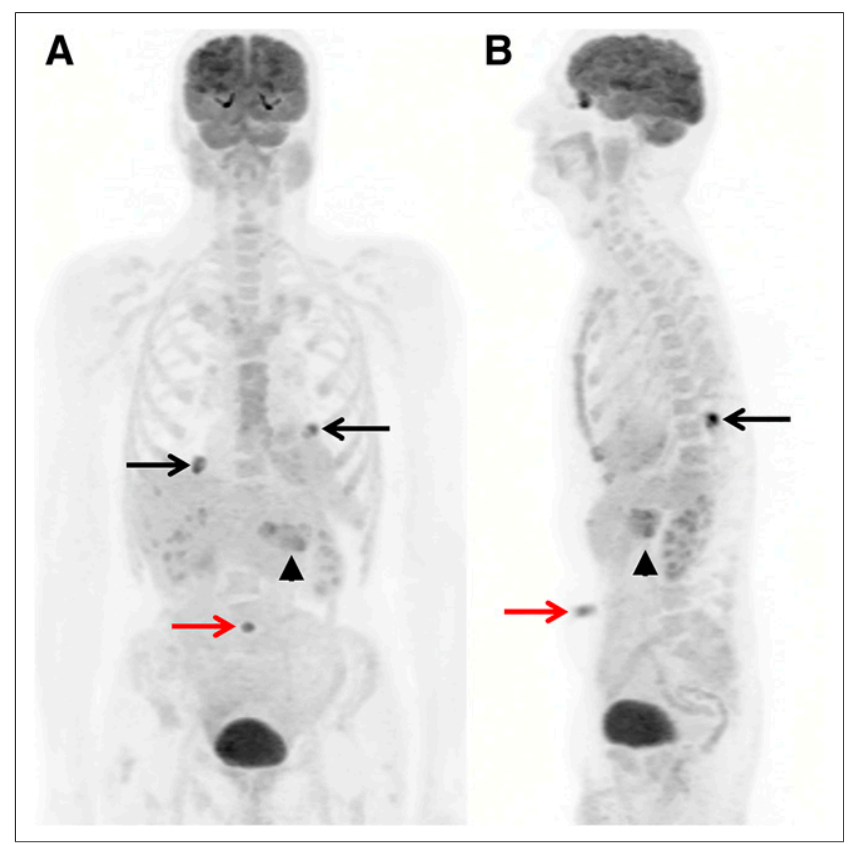

FIGURE 1. On torso PET/MRI survey, maximum-intensityprojection anterior $(A)$ and lateral $(B)$ views show tracer-avid lesion at umbilical region (red arrows) with SUV $\max$ of 7.1 and primary pancreatic tumor (arrowheads) with SUV max $_{\max }$ of 6.7. Two additional benign inflammatory lesions were noted bilaterally in lung bases (black arrows). Liver lesion with low ${ }^{18} \mathrm{~F}-\mathrm{FDG}$ uptake was not discernable on maximum-intensity projections. 


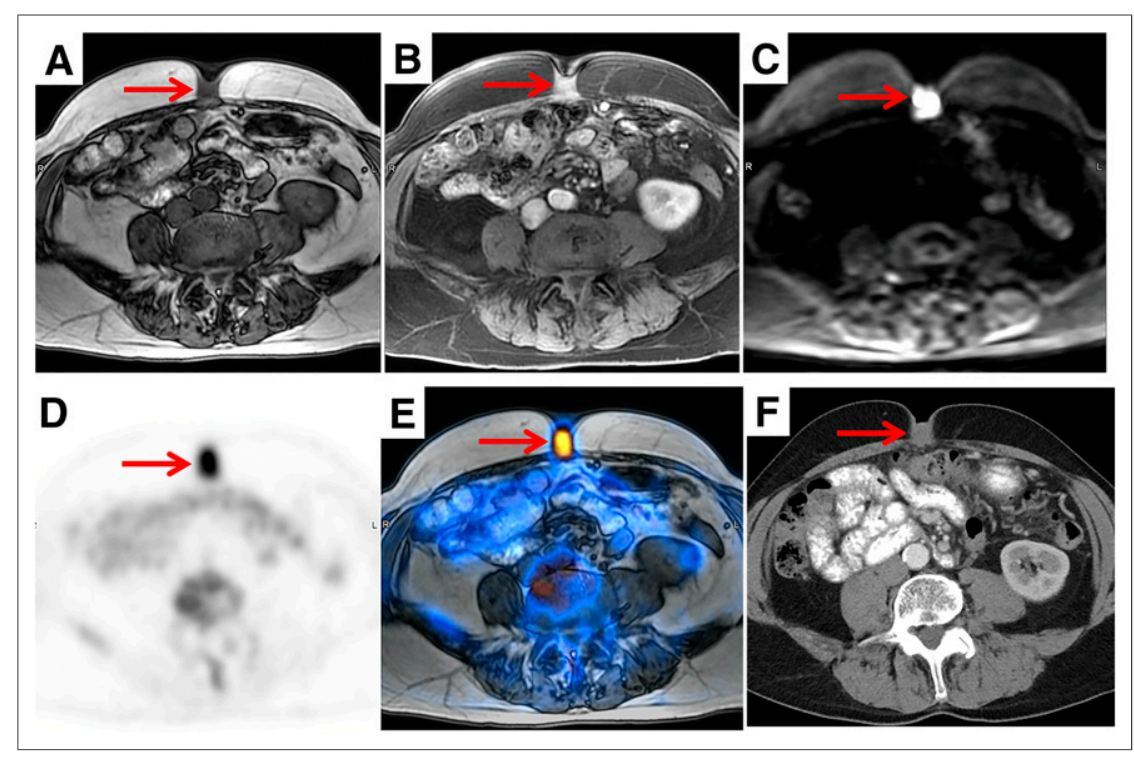

FIGURE 2. (A-C) Focused abdominal PET/MRI demonstrates 2.3-cm lesion (arrows) with low $T 1$ signal $(A)$, intense enhancement on postcontrast image $(B)$, and restricted diffusion on diffusion-weighted image $(C)$. (D and $E$ ) Lesion exhibited intense tracer uptake on PET (D) and PET/MRI (E) images. (F) This lesion was initially diagnosed clinically as umbilical fungal infection and was overlooked on contrast-enhanced abdominal CT study obtained at another institution.

of disseminated pancreatic cancer, FOLFIRINOX therapy was resumed.

\section{DISCUSSION}

The Sister Mary Joseph nodule represents an umbilical metastatic lesion that typically originates from gastrointestinal and gynecologic malignancies. The most common primary sites are stomach $(21 \%)$, ovary $(18 \%)$, colon $(12 \%)$, and pancreas $(9 \%)$. Other sites of rarer primaries include uterus, biliary tree, breast, and lung (1-2). The condition was named for Sister Mary Joseph, the surgical assistant to Dr. William Mayo of Mayo Clinic. She first noticed the association between an umbilical nodule and advanced intraabdominal malignancies while preparing the patient's skin before surgery.

The presence of the Sister Mary Joseph nodule is a sign of widespread abdominal or pelvic malignancy and usually indicates a poor prognosis (2). The tumor markers, including carbohydrate antigen 19-9, carcinoembryonic antigen, and carbohydrate antigen 125 , should be examined. The mechanism of tumor spread to the umbilicus has been postulated as direct invasion from the peritoneum through hematogenous or lymphatic pathways or along the embryonic remnants of the ligamentum teres or umbilical ligaments (3-4). In addition to metastasis, the differential diagnosis of a neoplastic umbilical nodule includes primary carcinoma, endometriosis, hernia, and granuloma, among others (5).

To our knowledge, this is the first report of identification of a Sister Mary Joseph nodule using PET/MRI. ${ }^{18} \mathrm{~F}-\mathrm{FDG}$ $\mathrm{PET} / \mathrm{MRI}$ is superior to $\mathrm{PET} / \mathrm{CT}$ in providing excellent soft-tissue delineation of the nodule on the conjunctive abdominal MRI.

\section{CONCLUSION}

${ }^{18} \mathrm{~F}-\mathrm{FDG}$ PET/MRI is a novel imaging tool in the diagnosis and staging of pancreatic adenocarcinoma. It provides an easier target for biopsy to establish the diagnosis of widespread pancreatic cancer with umbilical metastasis.

\section{DISCLOSURE}

No potential conflict of interest relevant to this article was reported.

\section{REFERENCES}

1. Yendluri V, Centeno B, Springett GM. Pancreatic cancer presenting as a Sister Mary Joseph's nodule: case report and update of the literature. Pancreas. 2007;34: 161-164.

2. Chiang $\mathrm{CH}$, Lin $\mathrm{MH}$. Sister Mary Joseph nodule associated with pancreatic adenocarcinoma. J Formos Med Assoc. 2015;114:92-93.

3. Métivier D Jr, Bonardel G, Rouquie D, et al. ${ }^{18} \mathrm{~F}$-FDG PET/CT imaging of Sister Mary Joseph's nodule. Clin Nucl Med. 2012;37:486-488.

4. Inanir S, Oksuzoglu K. FDG PET/CT imaging of calcified Sister Mary Joseph nodule. Clin Nucl Med. 2016;41:e458-e459.

5. Coll DM, Meyer JM, Mader M, et al. Imaging appearances of Sister Mary Joseph nodule. Br J Radiol. 1999;72:1230-1233. 